\title{
A771726, an anti-inflammatory drug, exerts an anticancer effect and reverses tamoxifen resistance in endocrine-resistant breast cancer cells
}

\author{
OU HUANG ${ }^{1 *}$, ZUOQUAN XIE $^{2 *}$, WEILI ZHANG ${ }^{3}$, YING LOU $^{1}$, YAN MAO $^{1}$, \\ HONGCHUN LIU ${ }^{2}$, MIN JIANG ${ }^{4}$ and KUNWEI SHEN ${ }^{1}$
}

\begin{abstract}
${ }^{1}$ Comprehensive Breast Health Centre, Ruijin Hospital, Shanghai Jiaotong University School of Medicine, Shanghai 200025;
${ }^{2}$ Division of Antitumor Pharmacology, Shanghai Institute of Materia Medica, Chinese Academy of Sciences, Shanghai 201203;

${ }^{3}$ Department of Gastroenterology, Xiangcheng People's Hospital, Suzhou, Jiangsu 215131; ${ }^{4}$ Department of Breast Surgery, The First Affiliated Hospital of Soochow University, Suzhou, Jiangsu 215006, P.R. China
\end{abstract}

Received February 24, 2014; Accepted May 21, 2014

DOI: $10.3892 /$ or.2014.3249

\begin{abstract}
A771726, an orally available anti-inflammatory agent, has been approved for the treatment of multiple sclerosis by diminishing entire inflammatory responses through multiple signaling pathways. Recently, a few emerging studies have focused on the potential application of A771726 in cancer therapy, less on the treatment of breast cancer and particularly on overcoming drug resistance in breast cancer. We report here for the first time the cytotoxic activity and drug resistance reversal of A771726 in acquired tamoxifen-resistant breast cancer cell line MCF-7/LCC9. We discovered that A771726 treatment showed antiproliferative activities in MCF-7/LCC9 cells, which were even more sensitive to A771726 than their parental tamoxifen-sensitive cells (MCF-7). A771726 also exerted pro-apoptotic activities and induced cell cycle arrest at the G1 phase. Notably, treatment of A771726 restored the sensitivity of MCF-7/LCC9 cells to tamoxifen. Western blot analysis revealed that A771726 decreased the phosphorylation level of Src, one key driver of tamoxifen resistance. Moreover, in order to comprehensively clarify the mechanisms of A771726 in anti-estrogen-resistant cells, we explored a genome-wide transcriptomic analysis, and showed that
\end{abstract}

Correspondence to: Professor Kunwei Shen, Comprehensive Breast Health Centre, Ruijin Hospital, Shanghai Jiaotong University School of Medicine, No. 197 Rui Jin Er Road, Shanghai 200025, P.R. China

E-mail: kunwei_shen@yeah.net

Dr Min Jiang, Department of Breast Surgery, The First Affiliated Hospital of Soochow University, No. 188 Shi Zi Road, Suzhou, Jiangsu 215006, P.R. China

E-mail: jiangmin1023@suda.edu.cn

*Contributed equally

Key words: A771726, endocrine resistance, tamoxifen, apoptosis, cell cycle, microarray analysis
A771726 could modulate multiple signaling pathways (e.g. cell cycle, apoptosis, MAPK, metabolism and p53 signaling pathway) and cellular processes (e.g. signal transduction, transcription and cell cycle). Overall, our results indicate that A771726 alone and the combination of A771726 with antiestrogens may be of therapeutic benefit for ER-positive and endocrine-resistant breast cancer.

\section{Introduction}

Breast cancer is the most commonly diagnosed cancer and the second leading cause of cancer-related mortality among women in the US (1). Breast cancer is a type of hormonedependent malignancy, and sustained exposure to estrogen is known to contribute to the development and progression of this disease (2). The biological effects of estrogen are mediated by the estrogen receptor (ER) through classic genomic and nongenomic actions (3). In the US, $75 \%$ of breast cancers express ER (4), and are classified as ER-positive breast cancer, thus corroborating the use of selective ER modulators (SERMs) as estrogen antagonists (5). Tamoxifen, the most commonly used SERM, has been accepted as the gold standard of endocrine therapy and has been utilized successfully both in neoadjuvant and adjuvant settings (6). However, a substantial proportion of breast cancer patients with localized disease and nearly all with advanced disease, despite an initial positive drug response, develop acquired tamoxifen resistance (7). To date, application of commercially available drugs to circumvent tamoxifen resistance remains sparse, and therefore, searching for novel pharmaceutical agents for re-sensitization to endocrine therapy is urgent.

A771726, the main active metabolite of leflunomide, also named teriflunomide, has been approved by the FDA for the treatment of multiple sclerosis (MS) (8). A771726 exerts antiinflammatory and immunomodulatory actions by inhibiting the activity of proliferating B- and T-cells through dihydroorotate dehydrogenase (DHODH) inhibition-dependent and -independent patterns (9). Although the precise underlying mechanisms remain elusive, A771726 has high plasma concentrations (10), 
a long elimination half-time (11), high treatment compliance (12) and multiple molecular targets (13), implying more possibilities for broader clinical applications, even beyond the treatment of MS.

Actually, A771726 has been reported to exert a potent anticancer effect in treating multiple human neoplasms (14-17). Notably, A771726 has been used as an agent with direct cytotoxicity to chemoresistant chronic lymphocytic leukemia (CLL) (18), and has also been utilized to enhance chemosensitization $(19,20)$ and assist targeting therapy $(21,22)$ in the treatment of other types of tumors. Further investigation on the application of A771726 in endocrine therapy for breast cancer has not yet been documented. Primary mechanisms of acquired resistance to tamoxifen in ER-positive breast cancer include deregulation of cell cycle and apoptotic machinery accompanied by altered modulation of various regulators, such as Bcl-2, p21, activation of receptor tyrosine kinase signaling leading to the increased activity of Src and MAPK pathways, and alteration of ER and ER co-regulators (23). In the present study, using an acquired endocrine-resistant cell model, MCF-7/LCC9, we investigated, for the first time, to our knowledge, the anticancer activity and tamoxifen-resistance reversal of A771726. Furthermore, we performed a comprehensive profiling analysis of A771726-regulated molecules in MCF-7/LCC9 cells and validated that A771726 may modulate extensive cellular processes and multiple signaling pathways that are critical for endocrine resistance. Here, our study provides new clues for the reversal of resistance to endocrine therapy and the potential clinical applications of A771726 in the management of tamoxifen resistance in breast cancer patients.

\section{Materials and methods}

Cell culture and drug treatment. MCF-7 and tamoxifen-resistant MCF-7/LCC9 cells were kindly provided by Dr Robert Clarke (Georgetown University Medical Centre, Washington, DC, USA). Cells were routinely cultured in complete medium with $5 \%$ fetal bovine serum (FBS) (Invitrogen, Carlsbad, $\mathrm{CA}, \mathrm{USA}$ ) at $37^{\circ} \mathrm{C}$ in $5 \% \mathrm{CO}_{2}$. 4-Hydroxytamoxifen (4-OHT) was purchased from Sigma-Aldrich (St. Louis, MO, USA), and A771726 was kindly provided by Cinkate Corporation (Oak Park, IL, USA). For the tamoxifen sensitivity analysis, 2,000 cells/well were plated in phenol red-free IMEM plus $5 \%$ charcoal dextran-treated FBS (Tissue Culture Biologicals, Los Alamitos, CA, USA) for $24 \mathrm{~h}$ and were then treated with a combination of A771726 and OHT for 7 days.

Sulforhodamine B (SRB) assay. Cells were seeded in 96-well plates (Corning, Acton, MA, USA) and fixed by trichloroacetic acid (Sinopharm Reagent, Shanghai, China). Dried cells were stained by SRB (Sigma-Aldrich), which then was solubilized by Tris-base (Sinopharm Reagent). The absorbance was read on an automated microplate reader (VERSAmax; Molecular Devices, Sunnyvale, CA, USA).

Clonogenic assay. Cells were plated at 500 cells/well in 6-well plates (Corning) and incubated with various concentrations of A771726 for 2 weeks. Then, the colonies were stained with $0.5 \%$ crystal violet and counted.
Flow cytometric assay. For cell apoptosis analysis, after treatment of A771726 for $48 \mathrm{~h}$, cells were detached and washed with cooled PBS. Cells were then re-suspended and stained by Annexin V and propidium iodide (PI) (BD Pharmingen, San Diego, CA, USA). Analysis was performed on a FACSCalibur analyzer (Becton-Dickinson, San Jose, CA, USA). With respect to cell cycle analysis, cells were harvested and fixed by $70 \%$ cooled ethanol, and then stained with PI (Sigma-Aldrich). Analysis was also carried out on a FACSCalibur analyzer.

Western blot assay. All cells were treated with A771726 for $72 \mathrm{~h}$. The protein extracts were separated by SDS-PAGE and transferred to NC membranes (Millipore, Bedford, MA, USA). Src and p-Src proteins were visualized by a chemiluminescence system (GE Healthcare, Piscataway, NJ, USA).

Microarray analysis. Briefly, samples were utilized to synthesize double-stranded complementary DNAs (cDNAs), which were labelled and hybridized to dual-channel mRNA microarray version 1.0 (CapitalBio Corp., Beijing, China) following the manufacturer's instructions. Data were selected by using a threshold of $>2$ - and $<-2$-fold change under false discovery rate $(\mathrm{FDR})$ protection $(\mathrm{P}<0.05)$.

Statistical analysis. Statistical significance was evaluated by the Student's t-test with GraphPad Prism software 5.0. P-values $<0.05$ were considered to indicate statistically significant results.

\section{Results}

A771726 inhibits the cell proliferation of endocrine-resistant breast cancer cells. To examine the biological effects on cell proliferation of tamoxifen-resistant cells and their sensitive counterparts, we treated the cells with different doses of A771726 and performed an SRB assay (Fig. 1A). Viability of the cells decreased in a dose- and time-dependent manner. Moreover, tamoxifen-resistant MCF-7/LCC9 cells were more sensitive to inhibition by this agent than the tamoxifen-sensitive MCF-7 cells; marked early antiproliferative effects were observed within a 24-h period in the MCF-7/LCC9 cells but not until $48 \mathrm{~h}$ in the MCF-7 cells, respectively. Lower $\mathrm{IC}_{50}$ values were observed for A771726 in the MCF-7/LCC9 cells (72 h post-treatment: $\mathrm{IC}_{50}=64.72 \mu \mathrm{M}$ in MCF-7/LCC9 and $122.79 \mu \mathrm{M}$ in MCF-7 cells).

A771726 suppresses the colony-forming ability of endocrine-resistant breast cancer cells. To further demonstrate the antiproliferative effects of A771726 on the growth of MCF-7/LCC9 cells, a colony formation assay was carried out. As shown in Fig. 1B, the colony numbers of MCF-7 and MCF-7/LCC9 cells were significantly decreased in dosedependent manner, and long-term treatment of A771726 at the concentration of $100 \mu \mathrm{M}$ almost completely diminished the colony formation capability. The results were consistent with that of the SRB assay further indicating that A771726 inhibits the in vitro proliferation of MCF-7/LCC9 cells.

A771726 induces apoptosis in endocrine-resistant breast cancer cells. We aimed to ascertain whether the cell growth 
A

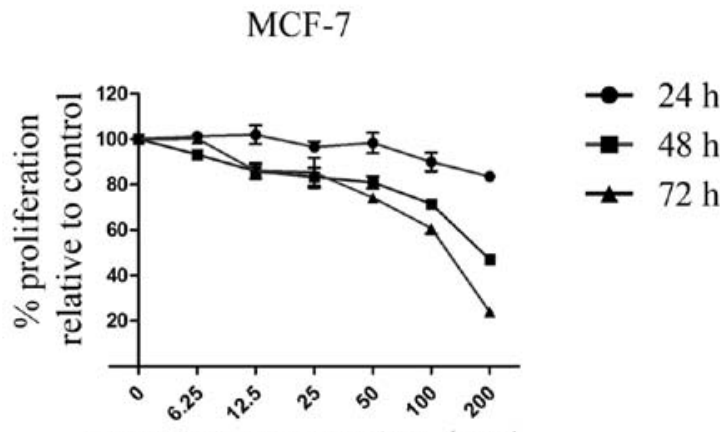

A77 1726 concentration $(\mu \mathrm{M})$
LCC9

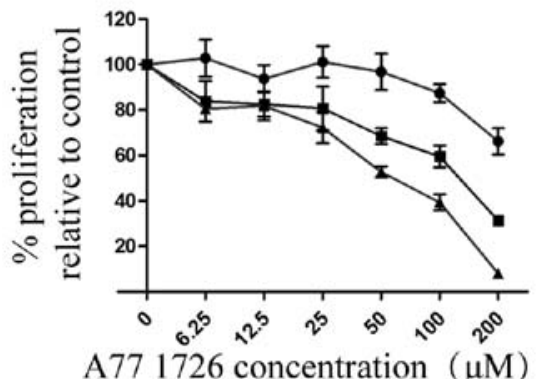

A77 1726 concentration ( $\mu \mathrm{M})$

B

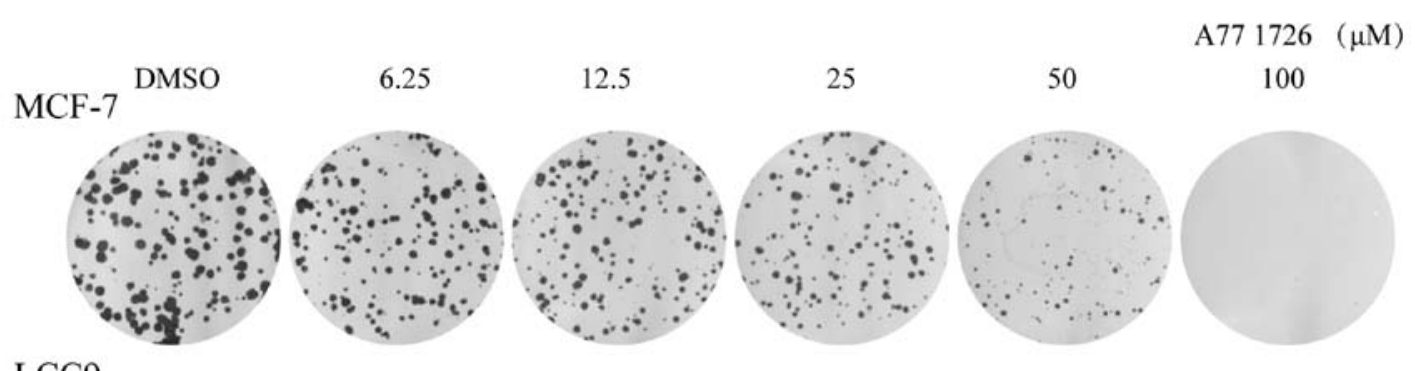

LCC9
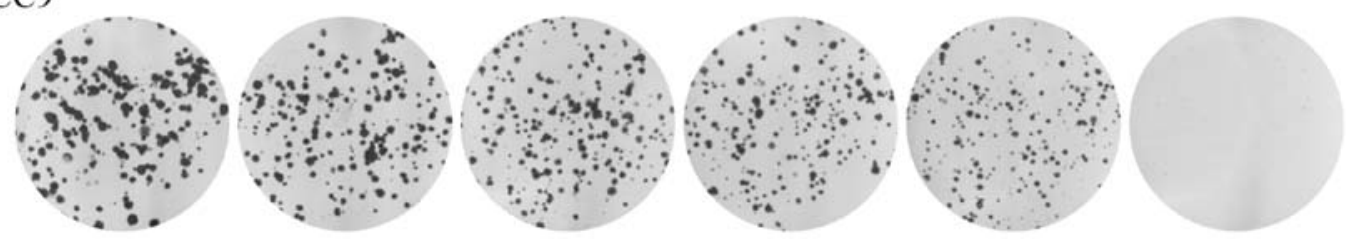

Figure 1. A771726 inhibits the proliferation of MCF-7/LCC9 cells. (A) MCF-7 and endocrine-resistant LCC9 cells were treated with varying concentrations of A771726, and cell proliferation and $\mathrm{IC}_{50}$ values were determined by SRB assay on day 1, 2 and 3. (B) A771726 suppressed colony formation. Cell were treated with A771726 and allowed to form colonies in fresh medium for 14 days. Photomicrographic differences are shown.

inhibition induced by A771726 was due to the induction of apoptosis in tamoxifen-resistant cells. Therefore, we incubated MCF-7/LCC9 and MCF-7 cells with A771726 for $48 \mathrm{~h}$. Our experiments showed that A771726 induced apoptosis in a dose-dependent manner in the endocrine-resistant and wild-type cells (Fig. 2A). When exposed to 50 and $100 \mu \mathrm{M}$ A771726, few MCF-7 cells were $\mathrm{PI}^{+}$or Annexin $\mathrm{V}^{+}$, indicating that low doses of A771726 did not induce significant apoptosis while the highest concentration of $200 \mu \mathrm{M}$ did. However, MCF-7/LCC9 cells were more sensitive to A771726; the lowest concentration of $50 \mu \mathrm{M}$ significantly increased the percentage of Annexin $\mathrm{V}^{+} / \mathrm{PI}^{+}$cells. Following treatment with higher doses of A771726 at 100 and $200 \mu \mathrm{M}$, the percentage of Annexin $\mathrm{V}^{+} /$ PI $^{-}$cells was increased from 4.48 to 18.63 and $27.85 \%$, as well as the percentage of Annexin $\mathrm{V}^{+} / \mathrm{PI}^{+}$cells from 3.06 to $37.05 \%$ at $200 \mu \mathrm{M}$, indicating $\mathrm{A} 771726$ at various concentrations induced apoptosis and necrosis in endocrine-resistant cell.

A771726 induces G1 phase arrest in endocrine-resistant breast cancer cells. In order to determine whether A771726 has a cell cycle arrest effect, MCF-7/LCC9 and MCF-7 cells treated with increasing doses of A771726 for $48 \mathrm{~h}$ were subjected to flow cytometric analysis. As shown in Fig. 2B, A771726 caused a dose-dependent accumulation of cells in the G1 phase fraction, while reducing cell accumulation in the $\mathrm{S}$ and $\mathrm{G} 2 / \mathrm{M}$ phases. The G1 phase fraction increased from 57.12 to $61.95 \%, 63.59$ and $77.74 \%$ at 50,100 and $200 \mu \mathrm{M} \mathrm{A771726} \mathrm{in} \mathrm{resistant} \mathrm{cells,}$ and from 54.30 to $62.60 \%, 68.78$ and $74.00 \%$ in wild-type cells at the same concentrations of A771726, accompanied by maximal decreases of $6.82 \%$ in the $\mathrm{S}$ phase and $13.79 \%$ in the G2/M phase in MCF-7/LCC9 cells and $10.53 \%$ in the $\mathrm{S}$ phase and $8.59 \%$ in the $\mathrm{G} 2 / \mathrm{M}$ phase in MCF-7 cells.

A771726 reverses tamoxifen resistance in endocrine-resistant breast cancer cells. To further examine the effects of A771726 on tamoxifen sensitivity, hormone-resistant MCF-7/LCC9 cells were treated with different doses of A771726 and tamoxifen (alone or in combination) for 7 days in estrogen-depleted media containing 5\% charcoal-stripped serum (CS). As shown in Fig. 3, cell viability assay recorded over a range of 4-OHT concentrations from 1 to $7 \mu \mathrm{M}$ confirmed that these cells indeed were resistant to OHT, compared with their sensitive counterparts (data not shown). After receiving A771726 plus OHT, dose-dependent increases in the sensitivity of MCF-7/LCC9 cells to OHT were observed. The $\mathrm{IC}_{50}$ value of $\mathrm{OHT}$ in the A771726-treated MCF-7/LCC9 cells was decreased to 7.0 and $5.9 \mu \mathrm{M}$ at 2.5 and $10 \mu \mathrm{M}$, indicating significant recovery of drug sensitivity.

A771726 inhibits Src activation in endocrine-resistant breast cancer cells. To investigate the mechanisms by which A771726 reverses the drug resistance of MCF-7/LCC9 cells, 
A



B


Figure 2. A771726 induces cell apoptosis and G1 phase arrest in MCF-7/LCC9 cells. (A) Flow cytometric analyses of Annexin V/PI staining showed the induction of apoptosis following treatment with different concentrations of A771726 for $48 \mathrm{~h}$. The lower right quadrant (Annexin $\mathrm{V}^{+} / \mathrm{PI}^{-}$) and upper right quadrant (Annexin $\mathrm{V}^{+} / \mathrm{PI}^{+}$) indicate the percentage of early apoptotic and late apoptotic cells. (B) MCF-7 and endocrine-resistant LCC9 cells were treated for $48 \mathrm{~h}$ with A771726 and percentages of cells in the three cell cycle phases were analyzed by flow cytometry. The percentage of cells in G1, S and G2/M phases of the cell cycle are shown. These results are from one representative experiment of three independent performances. PI, propidium iodide.

we analyzed the state of Src phosphorylation, one key driver of tamoxifen resistance (Fig. 4A). Although no change in total Src expression was detected between MCF-7 and MCF-7/LCC9 cells, our results revealed that the phosphorylation of $\mathrm{Src}$ (Tyr418) was increased in the resistant cells, both in complete medium with 5\% FBS and in phenol red-free medium with
5\% CS, and interestingly at a higher level in FBS. Following A771726 treatment, the Src phosphorylation was significantly suppressed at $100 \mu \mathrm{M} \mathrm{A771726}$ at $72 \mathrm{~h}$.

A771726 regulates global gene expression and multiple signaling pathways in endocrine-resistant breast cancer 
Table I. Differentially regulated genes in cell cycle, apoptosis and MAPK signaling pathways following A771726 treatment in MCF-7/LCC9 cells.

Signaling pathway

(oligo ID)

Gene symbol

Gene name

Fold-change

(LCC9-A771726

Cell cycle

H200008365

CDKN1A

Cyclin-dependent kinase inhibitor 1 (p21)

3.62

H300012891

CHEK1

H300020974

CCNE2

H200015433

$\mathrm{E} 2 \mathrm{~F} 2$

Serine/threonine-protein kinase Chk1

0.19

G1/S-specific cyclin-E2

0.41

Transcription factor E2F2

0.42

H200006619

MAD2L1

H200007139

CCNA2

Mitotic spindle assembly checkpoint protein MAD2A

0.44

Cyclin-A2

0.46

Apoptosis

$\begin{array}{ll}\text { opHsV0400006063 } & \text { CAPN2 } \\ \text { H300022026 } & \text { BAX } \\ \text { H300008499 } & \text { IRF1 } \\ \text { H300005750 } & \text { HSPA5 } \\ \text { H300019718 } & \text { BIRC5 } \\ \text { H200006652 } & \text { BCL2 }\end{array}$

MAPK

$\begin{array}{ll}\text { H300008649 } & \text { CACNG1 } \\ \text { H200000600 } & \text { DUSP4 } \\ \text { H200013671 } & \text { PLA2G3 } \\ \text { H300000118 } & \text { DUSP3 } \\ \text { H300019880 } & \text { ATF2 } \\ \text { H300016729 } & \text { MAP2K6 }\end{array}$

Calpain-2 catalytic subunit precursor

4.66

BAX protein

2.00

$\begin{array}{ll}\text { Interferon regulatory factor } 1 & 1.63\end{array}$

78 kDa glucose-regulated protein precursor (GRP 78) 0.43

Baculoviral IAP repeat-containing protein 5 (survivin) 0.49

Apoptosis regulator $\mathrm{Bcl}-2$

0.6011

Voltage-dependent calcium channel $\gamma$-1 subunit $\quad 4.85$

Dual specificity protein phosphatase $4 \quad 3.31$

Group 3 secretory phospholipase A2 precursor $\quad 2.88$

$\begin{array}{ll}\text { Dual specificity protein phosphatase } 3 & 2.14\end{array}$

Cyclic AMP-dependent transcription factor ATF-2 0.44

Dual specificity mitogen-activated protein kinase kinase $6 \quad 0.44$



Figure 3. A771726 reverses tamoxifen resistance in MCF-7/LCC9 cells. Cells were treated with a combination of increasing concentrations of OHT and A771726 or OHT alone for 7 days. Drug responses were determined by SRB assay. ${ }^{*} \mathrm{P}<0.05$. OHT, hydroxytamoxifen.

cells. To systematically gain further insight into the functional effects of A771726 in tamoxifen resistance reversal and other biological functions, we performed large-scale mRNA expression profiling following treatment of $100 \mu \mathrm{M}$ A771726 for $72 \mathrm{~h}$ in MCF-7/LCC9 cells. As shown in Fig. 4B, clustering results of all significantly regulated probes were demonstrated. Respectively, we identified 410 upregulated probes and 285 downregulated probes (Table I). To investigate the functional roles of the A771726-regulated genes, pathway analysis was performed, and genes modulated by A771726 in the MCF-7/LCC9 cells were significantly enriched in multiple KEGG pathways, represented by cell cycle, apoptosis, MAPK, metabolism and P53 signaling pathways (Fig. 4D). Gene ontology (GO) analysis showed that $53.84 \%$ of all differentially expressed genes were associated with biological process, $31.94 \%$ with molecular function and $14.23 \%$ with cellular component (Fig. 4C). The gene classes most significantly affected by A771726 were signal transduction and oxidation reduction in biological process, protein and nucleotide binding in molecular function and nucleus and cytoplasm in cell component (Fig. 4E).

\section{Discussion}

In the present study, we provide evidence that A771726 may have direct chemopreventive potential and resistance reversal when concurrently used in endocrine-resistant cells. We found that this anti-inflammatory agent is capable of suppressing the growth of MCF-7/LCC9 cells in vitro by inducing cell apoptosis and G1 phase arrest. Moreover, by analyzing A771726-regulated gene expression profiles in anti-estrogen-resistant cells, we demonstrated the multiple biological functions and signaling pathways that were involved.

Although recently approved by the FDA for use in the relapsing forms of MS in the US, A771726 has also been 
A

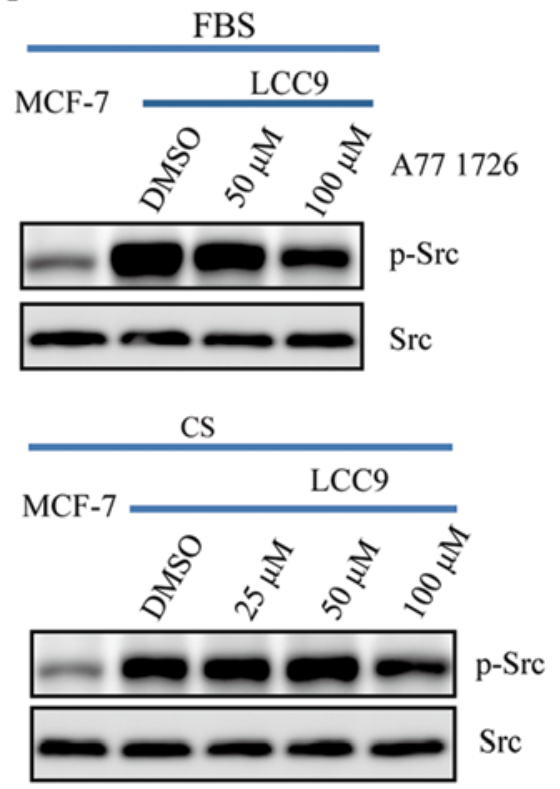

B



$\mathrm{D}$

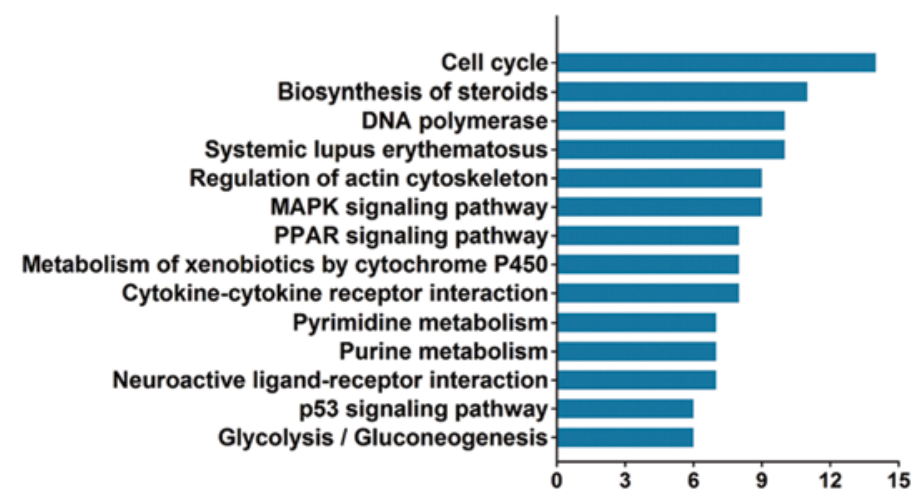

E

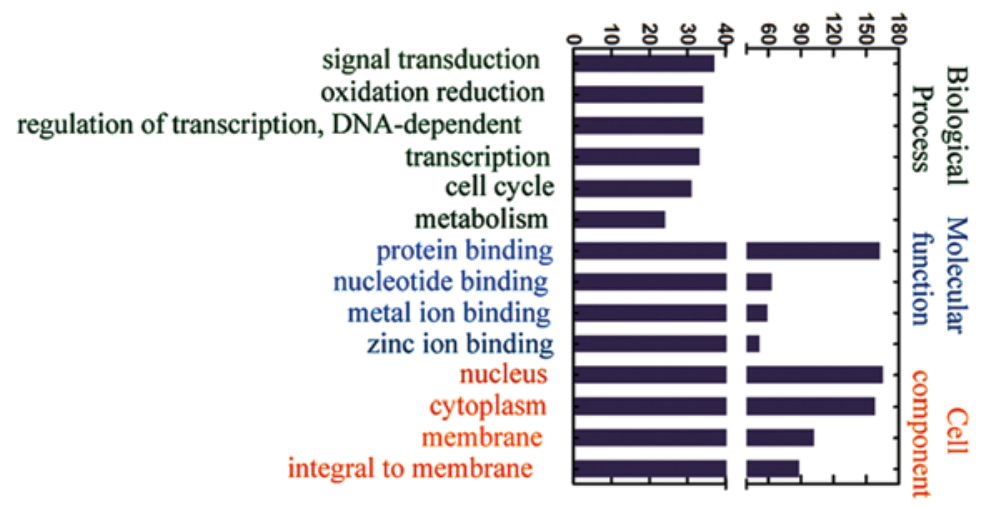

$\mathrm{C}$

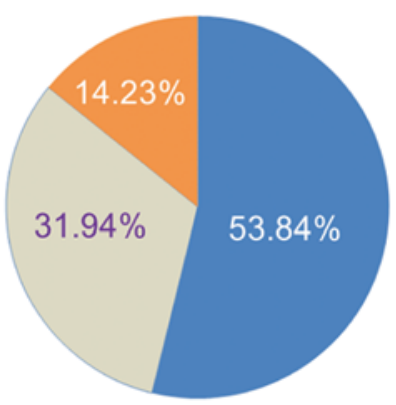

Biological process

Molecular function

Cellular component

Figure 4. A771726 suppresses Src phosphorylation and regulates global genes in MCF-7/LCC9 cells. (A) Cells were treated with A771726 for $72 \mathrm{~h}$ and were then subjected to western blotting. (B) Heat map of genes significantly upregulated and downregulated following A771726 treatment. (C) Fraction of deregulated genes in terms of three GO terms. (D) Representative signaling pathways significantly regulated by A771726. (E) Detailed distribution of total aberrantly expressed genes according to GO classification. GO, gene ontology.

investigated as a potential anticancer agent in a handful of human tumors (14-17,21). However, the lethal effect of A771726 in breast cancer has not been thoroughly investigated. In the present study, we report the potent cytotoxicity and attenuation of colony-forming activity of A771726 in sensitive ER-positive breast cancer cells (Fig. 1A and B). Notably, consistent with the direct anticancer effect in drug-resistance CLL (18), we also showed that A771726 led to cell death in endocrine-resistant cells (Fig. 1A and B). Moreover, we discovered that the tamoxifen-resistant MCF-7/LCC9 cells were more sensitive to A771726-mediated killing than the parental MCF-7 cells (Fig. 1A), indicating that resistance-related signals 
may be responsible for the differential response to A771726. Transcriptional repression or somatic deletion of CDKN1A (encoding p21), resulting in the alleviation of the inhibitory effect on CDK activity, contributes to decreased anti-estrogen sensitivity in breast cancer $(24,25)$. Although no change in Myc, a suppressor of p21 (24), was observed (data not shown), significant upregulation of p21 mRNA (Table I) was determined, leading to the inability of growth maintenance and retrieval of tamoxifen sensitivity.

Tamoxifen has the ability to elicit cell apoptosis via an ER-dependent and -independent manner, disturbing the balance between pro- and anti-survival functions (26). However, when endocrine resistance develops, cancer cells also lose the response to tamoxifen-induced apoptosis (27). Accumulating evidence suggests that the increased expression of anti-apoptotic Bcl-2 family members, such as Bcl-2 and $\mathrm{Bcl}-\mathrm{xL}$, and functional defect of pro-apoptotic members, such as BAK and BIK, contribute to the emergence of attenuated responses to tamoxifen (23). In the present study, A771726 treatment in resistant cells induced downregulation of antiapoptotic Bcl-2 by $40 \%$ and upregulation of the pro-apoptotic Bax (Table I), leading to an increase in the BAX:Bcl-2 ratio, shifting the balance towards cell death (28) and restoring the tamoxifen-induced apoptosis in MCF-7/LCC9 cells (29). In addition, compared with the sensitive controls, MCF-7/LCC9 cells expressed lower IRF1 (30), whose enhanced expression by A771726 (by $\sim 60 \%$ ) may mediate cell death by inversing the balance of Bcl-2 family members and downregulating the inhibitor of apoptosis, survivin (Table I) (31), thus, determining the cell fate decision to again undergo drug apoptosis. Interestingly, we also revealed that autophagy may be involved in A771726-induced sensitivity restoration by inhibiting expression of GRP78 (Table I), which could activate mTORregulated dependent pro-survival autophagy with increased LC3-II and decreased p62 in induction of anti-estrogen resistance (32). Overall, our results showed that A771726 could recover the deregulated apoptosis and autophagy in tamoxifenresistant cells via modulating multiple signals involved in the determination of cell fate.

In addition to the disruption of cell death pathways, cell cycle perturbation by A771726, consistent with data in the treatment of multiple myeloma cells (19), could also be observed in endocrine-resistant cells. Cyclin E2 is induced in the late $\mathrm{G} 1$ phase and activates cyclin-dependent kinase CDK2, leading to inactivation of $\mathrm{pRB}$ and activation of E2F transcription factors to promote entry into $\mathrm{S}$ phase (33). However, in the development of impaired tamoxifen responsiveness, aberrant regulation of several cell cycle regulators, such as cyclin E2 and E2F2, also anti-estrogen targets, was found to be associated with the inability of induction of G1 phase-specific cell cycle arrest and the consequent reduction in cancer growth $(34,35)$. Interestingly, our genome-wide analysis in MCF-7/LCC9 cells showed that A771726 significantly decreased the expression of cyclin E2 and E2F2 (Table I) and CDK2 by $25 \%$, accompanied by the upregulation of the antagonist of CDK inhibitor, p21 (33), contributing to the delay of cell cycle progression from the G1 to the S phase (Fig. 2B) and enhancement of tamoxifen-induced growth inhibition (Fig. 3). Moreover, downregulation of GRP78 (Table I), also a key driver of tamoxifen resistance (32), may be involved in G1 arrest (36).
Prior experiments have demonstrated that the resistant cell line MCF-7/LCC9 has an elevated level of Src phosphorylation (37), which was also observed in the present study, regardless of whether the medium contained estrogen or not (Fig. 4A). Pharmacological inhibition of Src, like that in A771726 treatment in resistant cells, may be effective in preventing the emergence of tamoxifen resistance (38). For the first time, we demonstrated the global pattern of cellular functions and signaling pathways of A771726 targets using GO and pathway analysis. As for pathway mapping, we revealed that multiple oncogenic signaling pathways, cell cycle, apoptosis, MAPK, metabolism and p53 pathway, were regulated by A771726 (Fig. 4D), which have been reported to contribute to acquisition of tamoxifen resistance $(23,39)$. Although precise underlying regulation patterns of the biological processes have not yet been uncovered, A771726 modulated signal transduction, oxidation reduction, transcription, cell cycle and metabolism (Fig. 4E), consistent with that in the pathway analysis. Moreover, the A771726-related gene products were mainly located in the nuclei, indicating its involvement in the regulation of transcription.

In summary, to the best of our knowledge for the first time, we demonstrated the mechanistic evidence for the potent anticancer effect and tamoxifen resistance reversal by A771726 in endocrine-resistant MCF-7/LCC9 cells. Our findings demonstrated that A771726 mediated these effects through modulation of extensive related signaling pathways and cellular processes. The data provide support for the potential clinical applications of A771726 for ER-positive breast cancer patients with poor response to endocrine therapy.

\section{Acknowledgements}

The present study was supported by the National Natural Science Foundation of China (nos. 81202549, 81202088, 81302147 and 81302145 ), the National Science and Technology Major Project of the Ministry of Science and Technology of China (no. 2012ZX09301001-007 to M.G.), the Leading Academic Discipline Project of Shanghai Municipal Education Commission (J50208), the Cancer Foundation of China (0901), the Science and Technology Commission of Shanghai Municipality (09411961400), and the Shanghai Charity Cancer Research Centre.

\section{References}

1. Siegel R, Ma J, Zou Z and Jemal A: Cancer statistics, 2014. CA Cancer J Clin 64: 9-29, 2014.

2. Chlebowski RT, Manson JE, Anderson GL, et al: Estrogen plus progestin and breast cancer incidence and mortality in the Women's Health Initiative Observational Study. J Natl Cancer Inst 105: 526-535, 2013.

3. McDonnell DP and Norris JD: Connections and regulation of the human estrogen receptor. Science 296: 1642-1644, 2002.

4. Brouckaert O, Paridaens R, Floris G, Rakha E, Osborne K and Neven P: A critical review why assessment of steroid hormone receptors in breast cancer should be quantitative. Ann Oncol 24: 47-53, 2013

5. Pinkerton JV and Thomas S: Use of SERMs for treatment in postmenopausal women. J Steroid Biochem Mol Biol 142C: 142-154, 2014.

6. Goncalves R, Ma C, Luo J, Suman V and Ellis MJ: Use of neoadjuvant data to design adjuvant endocrine therapy trials for breast cancer. Nat Rev Clin Oncol 9: 223-229, 2012. 
7. Early Breast Cancer Trialists' Collaborative Group (EBCTCG): Effects of chemotherapy and hormonal therapy for early breast cancer on recurrence and 15-year survival: an overview of the randomised trials. Lancet 365: 1687-1717, 2005.

8. Ali R, Nicholas RS and Muraro PA: Drugs in development for relapsing multiple sclerosis. Drugs 73: 625-650, 2013.

9. Oh J and O'Connor PW: Teriflunomide. Neurol Clin Pract 3: 254-260, 2013.

10. Tallantyre E, Evangelou N and Constantinescu CS: Spotlight on teriflunomide. Int MS J 15: 62-68, 2008.

11. Wiese MD, Rowland A, Polasek TM, Sorich MJ and O'Doherty C: Pharmacokinetic evaluation of teriflunomide for the treatment of multiple sclerosis. Expert Opin Drug Metab Toxicol 9: 1025-1035, 2013.

12. Oh J and O'Connor PW: Safety, tolerability, and efficacy of oral therapies for relapsing-remitting multiple sclerosis. CNS Drugs 27: 591-609, 2013.

13. Oh J and O'Connor PW: An update of teriflunomide for treatment of multiple sclerosis. Ther Clin Risk Manag 9: 177-190, 2013.

14. Zhu S, Yan X, Xiang Z, Ding HF and Cui H: Leflunomide reduces proliferation and induces apoptosis in neuroblastoma cells in vitro and in vivo. PLoS One 8: e71555, 2013.

15. Hail N Jr, Chen P, Kepa JJ and Bushman LR: Evidence supporting a role for dihydroorotate dehydrogenase, bioenergetics, and p53 in selective teriflunomide-induced apoptosis in transformed versus normal human keratinocytes. Apoptosis 17: 258-268, 2012.

16. Ringshausen I, Oelsner M, Bogner C, Peschel C and Decker T: The immunomodulatory drug Leflunomide inhibits cell cycle progression of B-CLL cells. Leukemia 22: 635-638, 2008.

17. Hail N Jr, Chen P and Bushman LR: Teriflunomide (leflunomide) promotes cytostatic, antioxidant, and apoptotic effects in transformed prostate epithelial cells: evidence supporting a role for teriflunomide in prostate cancer chemoprevention. Neoplasia 12: 464-475, 2010

18. Dietrich S, Krämer $\mathrm{OH}, \mathrm{Hahn} \mathrm{E}$, et al: Leflunomide induces apoptosis in fludarabine-resistant and clinically refractory CLL cells. Clin Cancer Res 18: 417-431, 2012.

19. Baumann P, Mandl-Weber S, Völkl A, et al: Dihydroorotate dehydrogenase inhibitor A771726 (leflunomide) induces apoptosis and diminishes proliferation of multiple myeloma cells. Mol Cancer Ther 8: 366-375, 2009.

20. Uckun FM: Rationally designed anti-mitotic agents with proapoptotic activity. Curr Pharm Des 7: 1627-1639, 2001.

21. White RM, Cech J, Ratanasirintrawoot S, et al: DHODH modulates transcriptional elongation in the neural crest and melanoma. Nature 471: 518-522, 2011.

22. Somnay Y, Chen $\mathrm{H}$ and Kunnimalaiyaan M: Synergistic effect of pasireotide and teriflunomide in carcinoids in vitro. Neuroendocrinology 97: 183-192, 2013.

23. Musgrove EA and Sutherland RL: Biological determinants of endocrine resistance in breast cancer. Nat Rev Cancer 9: 631-643, 2009.

24. Mukherjee S and Conrad SE: c-Myc suppresses p21 $1^{\mathrm{WAF} 1 / \mathrm{CIP} 1}$ expression during estrogen signaling and antiestrogen resistance in human breast cancer cells. J Biol Chem 280: 17617-17625, 2005.
25. Abukhdeir AM, Vitolo MI, Argani P, et al: Tamoxifen-stimulated growth of breast cancer due to p21 loss. Proc Natl Acad Sci USA 105: 288-293, 2008.

26. Riggins RB, Bouton AH, Liu MC and Clarke R: Antiestrogens, aromatase inhibitors, and apoptosis in breast cancer. Vitam Horm 71: 201-237, 2005

27. Razandi M, Pedram A, Jordan VC, Fuqua S and Levin ER: Tamoxifen regulates cell fate through mitochondrial estrogen receptor beta in breast cancer. Oncogene 32: 3274-3285, 2013.

28. Martin LA and Dowsett M: BCL-2: a new therapeutic target in estrogen receptor-positive breast cancer? Cancer Cell 24: 7-9, 2013.

29. Nehra R, Riggins RB, Shajahan AN, Zwart A, Crawford AC and Clarke R: BCL2 and CASP8 regulation by NF- $\kappa$ B differentially affect mitochondrial function and cell fate in antiestrogen-sensitive and -resistant breast cancer cells. FASEB J 24: 2040-2055, 2010.

30. Ning Y, Riggins RB, Mulla JE, Chung H, Zwart A and Clarke R: IFN $\gamma$ restores breast cancer sensitivity to fulvestrant by regulating STAT1, IFN regulatory factor $1, N F-\kappa B$, BCL2 family members, and signaling to caspase-dependent apoptosis. Mol Cancer Ther 9: 1274-1285, 2010.

31. Schwartz JL, Shajahan AN and Clarke R: The role of interferon regulatory factor-1 (IRF1) in overcoming antiestrogen resistance in the treatment of breast cancer. Int J Breast Cancer 2011: 912102, 2011.

32. Cook KL, Shajahan AN, Wärri A, Jin L, Hilakivi-Clarke LA and Clarke R: Glucose-regulated protein 78 controls cross-talk between apoptosis and autophagy to determine antiestrogen responsiveness. Cancer Res 72: 3337-3349, 2012.

33. Geng Y and Sicinski P: Differences in regulation and function of E-cyclins in human cancer cells. Cell Cycle 12: 1165, 2013.

34. Caldon CE, Sergio CM, Kang J, et al: Cyclin E2 overexpression is associated with endocrine resistance but not insensitivity to CDK2 inhibition in human breast cancer cells. Mol Cancer Ther 11: 1488-1499, 2012

35. Miller TW, Balko JM, Fox EM, et al: ER $\alpha$-dependent E2F transcription can mediate resistance to estrogen deprivation in human breast cancer. Cancer Discov 1: 338-351, 2011.

36. Lin JA, Fang SU, Su CL, et al: Silencing glucose-regulated protein 78 induced renal cell carcinoma cell line G1 cell-cycle arrest and resistance to conventional chemotherapy. Urol Oncol 32: 29.e1-29.e11, 2014

37. Jiang $M$, Huang $O$, Zhang $X$, et al: Curcumin induces cell death and restores tamoxifen sensitivity in the antiestrogen-resistant breast cancer cell lines MCF-7/LCC2 and MCF-7/LCC9. Molecules 18: 701-720, 2013.

38. Hiscox S, Jordan NJ, Smith C, et al: Dual targeting of Src and ER prevents acquired antihormone resistance in breast cancer cells. Breast Cancer Res Treat 115: 57-67, 2009.

39. Martinez-Outschoorn UE, Goldberg A,Lin Z, et al: Anti-estrogen resistance in breast cancer is induced by the tumor microenvironment and can be overcome by inhibiting mitochondrial function in epithelial cancer cells. Cancer Biol Ther 12: 924-938, 2011. 